\title{
Pragmatic abilities of children with Williams syndrome: a longitudinal examination
}

\author{
Angela E. John ${ }^{1,2}{ }^{*}$, Lauren A. Dobson ${ }^{1}$, Lauren E. Thomas ${ }^{1}$ and Carolyn B. Mervis ${ }^{1}$ \\ ${ }^{1}$ Department of Psychological and Brain Sciences, University of Louisville, Louisville, KY, USA \\ ${ }^{2}$ Medical Investigation of Neurodevelopmental Disorders Institute, University of California Davis, Sacramento, CA, USA
}

Edited by:

Daniela Plesa Skwerer, Boston

University, USA

Reviewed by:

Teresa Mitchell, University of Massachusetts Medical School, USA

Vesna Stojanovik, University of

Reading, UK

${ }^{*}$ Correspondence:

Angela E. John, Medical Investigation of Neurodevelopmental Disorders Institute, University of California

Davis, 2825 50th Street, Room 2101, Sacramento, CA 95817, USA.

e-mail: angela.john@ucdmc. ucdavis.edu

\begin{abstract}
Prior research has indicated that pragmatics is an area of particular weakness for individuals with Williams syndrome (WS). To further address this aspect of the WS social phenotype, we used an individual differences approach to consider both cross-sectional and longitudinal relations among different pragmatic abilities for 14 children with WS, taking into account individual differences in non-verbal reasoning abilities. We also considered the relations between pragmatic abilities and expressive vocabulary ability. Participants were tested at two time points: as 4-year-olds during a 30-min play session with their mothers (Time 1) and an average of 5.87 years later during a one-on-one conversation with a familiar researcher (Time 2). Children's intellectual and expressive vocabulary abilities were assessed at both time points. Results indicated that the ability to verbally contribute information beyond what was required in response to a question (ExtendQ) was significantly related to the ability to verbally contribute new information in the absence of a question (ExtendS) both at age 4 years and during primary school. At age 4 , both the ability to pair verbalizations with eye contact in triadic interactions (secondary intersubjectivity) and expressive vocabulary ability were related to both ExtendQ and ExtendS. Finally, both ExtendQ and the ability to pair verbalizations with eye contact (intersubjectivity) at age 4 years predicted ExtendQ at age 9-12 years. The theoretical implications of our findings and the importance of early pragmatic language intervention for children who have WS are discussed.
\end{abstract}

Keywords: Williams-Beuren syndrome, conversation, social communication, pragmatics, longitudinal, intellectual disability

\section{INTRODUCTION}

Williams syndrome (WS) is a complex neurodevelopmental disorder resulting from a hemideletion of 26 genes on chromosome $7 q 11.23$ (Hillier et al., 2003). The prevalence of WS is estimated to be 1 in 7500 live births (Strømme et al., 2002) with both genders equally likely to be affected (American Academy of Pediatrics Committee on Genetics, 2001). Like most genetic syndromes, WS is associated with a specific physical and medical phenotype which includes dysmorphic facial features, heart disease (most commonly supravalvar aortic stenosis), connective tissue abnormalities, failure to thrive, and growth deficiency (Morris, 2006). The majority of children with WS demonstrate developmental delay that typically leads to mild to moderate intellectual disability or learning difficulties, although some individuals have low average to average intelligence. In addition, WS is associated with a specific cognitive profile characterized by relative strengths in verbal short-term memory and the structural and concrete vocabulary components of language accompanied by considerable weakness in visuospatial construction (Udwin and Yule, 1991; Mervis et al., 2000; Mervis and Morris, 2007).

Williams syndrome has drawn considerable attention from researchers and the general public due to the unique behavioral profile associated with this disorder. Individuals with WS demonstrate a considerable amount of interest in others (Klein-Tasman and Mervis, 2003; Mervis et al., 2003; Klein-Tasman et al., 2011) and are often described as outgoing and talkative and never going unnoticed in a group (Dilts et al., 1990; Fryns et al., 1991; Gosch and Pankau, 1997; Dykens and Rosner, 1999). These behavioral characteristics are likely a significant contributor to some authors' characterization of the WS social phenotype as the opposite of the autism social phenotype (e.g., Cowley, 2003; Levy et al., 2011). However, despite their sociable nature, individuals with WS have considerable difficulty navigating the surrounding world of people. Children with WS are delayed in the development of the ability to understand another person's perspective or theory of mind, an impairment that is also characteristic of the autism social phenotype (Tager-Flusberg et al., 1997; Tager-Flusberg and Sullivan, 2000; John and Mervis, 2009). In addition, individuals with WS have difficulty establishing and maintaining peer relationships (e.g., Davies et al., 1998; Sullivan et al., 2003) and most adults are socially isolated and do not typically engage in social interactions with peers (Udwin, 1990).

Difficulties with socio-communicative abilities likely contribute to these problems. To date, there have been no published studies of individuals with WS examining the stability of individual differences for the same type of pragmatic ability across time, and the only published study that addressed relations between pragmatic abilities and vocabulary abilities in individuals with WS (John et al., 2009) was cross-sectional and focused on receptive vocabulary rather than expressive. In the present study, to further 
understand the WS social phenotype, we considered whether individual differences in verbally contributing new information within a social interaction were related to the child's rate of pairing verbalizations with eye contact in triadic interactions and/or the child's expressive vocabulary ability, beyond the levels expected as a function of the children's non-verbal intellectual abilities. Children participated in this study twice, once as preschoolers and once approximately 6 years later, during primary school. Thus, we also were able to examine the relative stability of individual differences in children's verbal contribution of new information to a social interaction during the preschool and primary school-age periods and whether pairing verbalizations with eye contact and/or expressive vocabulary ability at age 4 years predicted the ability to verbally contribute new information during the primary school years beyond what would be expected given the children's non-verbal intellectual abilities.

\section{SOCIO-COMMUNICATIVE AND PRAGMATIC LANGUAGE ABILITIES OF INDIVIDUALS WITH WILLIAMS SYNDROME}

Over the last decade, a considerable amount of research has been dedicated toward understanding the intricacies of social cognitive and pragmatic development in individuals with WS. This body of literature stemmed from researchers' desire to understand why, despite their sociable nature and relative strength in the concrete and structural aspects of language, individuals with WS experienced so much difficulty establishing and maintaining peer relationships. The findings from these studies indicate that pragmatic difficulties are present across the life span.

Early in development, children with WS demonstrate delay in the emergence of joint attention ability - the ability to coordinate one's attention between a person and an object or event of mutual interest - not only relative to chronological age (CA) but also relative to language ability (Mervis and Bertrand, 1993, 1997; Mervis et al., 2003). In addition, young children with WS are significantly less likely to engage in triadic joint attention than are either mentalage matched typically developing (TD) children (Laing et al., 2002) or children with Down syndrome (DS) matched on CA, developmental quotient (DQ), and expressive vocabulary size (Rowe et al., 2005). Findings from two studies focused on the performance of toddlers and preschoolers with WS on a semi-structured play-based assessment (Autism Diagnostic Observation ScheduleGeneric, Module 1; Lord et al., 1999) indicated that approximately half of the participants in each study did not clearly integrate eye contact to reference an out-of-reach object to their communicative partner and the majority of participants did not integrate eye contact or vocalizations with acts of showing objects (Klein-Tasman et al., 2007; Lincoln et al., 2007). Finally, despite having significantly higher DQs, preschoolers with WS have significantly more difficulty inferring the communicative intent behind pointing and eye gaze gestures than do CA-matched preschoolers with DS (John and Mervis, 2010).

The development of joint attention has been argued to demonstrate the child's recognition of people as intentional agents (e.g., Tomasello, 1995; Thompson, 2006). Furthermore, for TD children, early triadic joint attention ability has been shown to predict later language development and the development of the ability to understand another person's perspective or theory of mind (e.g.,
Tomasello, 1995; Baldwin and Moses, 1996; Charman et al., 2000, 2003). As discussed by John et al. (2009), successful communication is partially dependent on the ability to take another person's perspective. Given the relation between early triadic joint attention abilities and later theory of mind in typical development, it is likely that the early impairments in triadic joint attention evidenced by children with WS are a contributing factor to their later impairments in pragmatics. While this hypothesis has not been tested directly, empirical findings documenting pragmatic difficulties during the adolescent and adult years and the significant association between pragmatic language ability and theory of mind for individuals with WS (e.g., Jones et al., 2000; Laws and Bishop, 2004; John and Mervis, 2009) are consistent with this hypothesis.

Several research groups have addressed the general pragmatic abilities of individuals with WS beyond the preschool years using parental responses on the Children's Communication Checklist (CCC; Bishop, 1998) or the CCC-2 (Bishop, 2002). Results have indicated that individuals with WS demonstrate particular difficulty with the use of stereotyped phrases, inappropriate initiation of conversations, and overdependence on context to interpret what was said to them (Laws and Bishop, 2004; Peregrine et al., 2005; Philofsky et al., 2007; Harmon et al., 2009). Some of these difficulties are comparable to those evidenced by children with autism; Philofsky et al. (2007) reported that school-age children with WS and CA-matched children with autism evidenced similar levels of impairment on the Inappropriate Initiation and Use of Context scales of the CCC-2. Klein-Tasman et al. (2011) administered the Social Responsiveness Scale (SRS; Constantino and Gruber, 2005) to the parents of eighty-two 4 - to 16-year-olds with WS and to the teachers of 49 of the children. Although the mean $T$ score on the Social Motivation subscale was in the average range for TD children based on both parental and teacher report, mean $T$ scores were in the mild to severe difficulty range for the remaining subscales (Social Awareness, Social Cognition, Social Communication, and Autistic Mannerisms), indicating considerable difficulty with many components of reciprocal social reciprocity.

In addition, several researchers have directly examined the conversational abilities of individuals with WS. Udwin and Yule (1990) collected $30 \mathrm{~min}$ conversations with a researcher for 43 children with WS (mean CA $=11.1$ years). The authors found that $37 \%$ of the participants met their criteria for hyper-verbal speech (fluent speech including an excessive number of stereotyped phrases or idioms, over-familiarity, introduction of irrelevant personal experiences, and perseverative responding). More recently, Jones et al. (2000) examined the spontaneous use of social language during a Biographical Interview task, which involved asking each participant questions about his or her family, activities, and interests. The authors reported data for adolescents and adults with WS ( $n=10$; mean $\mathrm{CA}=15.8$ years), CA- and IQ-matched individuals with DS ( $n=10$; mean $\mathrm{CA}=15.1$ years), and TD children matched on mental-age ( $n=8$; mean $\mathrm{CA}=6.5$ years). The number of interview questions answered by the three groups did not differ statistically. However, the WS group used significantly more evaluative devices (descriptions of affective states, evaluative comments, empathic markers, and character speech) than did either comparison group. Jones et al. also noted that the participants 
with WS often asked the interviewer personal questions and perseverated even when the interviewer tried to redirect them.

Stojanovik (2006) compared the pragmatic language abilities of five children with WS (mean $\mathrm{CA}=9.17$ years) to those of eight children with specific language impairment (SLI) matched for receptive vocabulary and grammatical ability (mean $\mathrm{CA}=10.58$ years) and nine $\mathrm{TD}$ children (mean $\mathrm{CA}=8.67$ years) during a semi-structured conversation. The children with WS were significantly less likely than were the children in either comparison group to add information to the conversation beyond that explicitly requested by their conversational partner. In addition, regardless of whether the researcher asked for information or clarification, the responses of the children with WS were less likely to be adequate than were the responses of either the children with SLI or the TD children. More specifically, the WS group was more likely to provide too little information or to misinterpret what the researcher had meant.

Finally, John et al. (2009) used a barrier listener-role referential communication task to examine the ability of children with WS ( $n=57$; mean CA $=9.24$ years) to verbalize message inadequacy. In this task, a researcher instructed the children to place a smaller picture on a larger scene. Although the children performed well when the researcher's instructions were adequate, they had considerable difficulty when the researcher provided inadequate instructions (i.e., the requested picture was not available, the instruction was ambiguous, or the instruction contained vocabulary that the child did not understand). Children verbally indicated that a problem was encountered less than half of the time on average and most of their verbalizations were either too vague for the researcher to determine the nature of the problem or indicated the wrong problem. Children's ability to verbalize message inadequacy was related to CA and theory of mind ability.

Given the findings of the studies examining the conversational abilities of individuals with WS conducted to date, it is not surprising that the WS social phenotype includes difficulties establishing and maintaining friendships. Even though individuals with WS demonstrate a sociable nature and are interested in interacting with others, tendencies to use stereotyped and perseverative utterances, to provide too little information or to misinterpret what their communicative partner meant, and to be less likely to contribute new information to the interaction likely serve as serious roadblocks in social interactions with peers.

While progress has been made in describing the pragmatic language abilities of individuals with WS across the lifespan, much remains to be understood. For example, to date there have been no studies reported that examined the stability of individual differences in the pragmatic language abilities of people with WS across time. In addition, although Fidler et al. (2007) have hypothesized that early difficulties with secondary intersubjectivity (relating/connecting to other people in triadic interactions) play a key role in the development of the WS phenotype, the question of whether secondary intersubjectivity ability is related to concurrent pragmatic language abilities or predicts later pragmatic language abilities in individuals with WS has not been addressed empirically. The purpose of the present study was to begin to address these gaps in the literature. The same types of pragmatic language data and expressive vocabulary ability data were collected on a group of children with WS at two time points. At Time 1, when the children were 4 years old, providing new information to a verbal interaction beyond what was explicitly requested (both in response to a question and in the absence of a question) was assessed during a 30-min play session with their mothers. An average of 5.87 years later (Time 2), the same pragmatic variables were assessed during a 7-min one-on-one conversation with a familiar researcher. Expressive vocabulary and non-verbal intellectual ability were assessed at both time points using standardized measures. Finally, the pairing of verbalizations with eye contact during the play session at Time 1 (a measure of secondary intersubjectivity) was also assessed.

We addressed several research questions. Our first set of questions was cross-sectional and was considered separately for Time 1 (preschool) and Time 2 (primary school). In particular, we sought to determine if there were significant relations among individual differences in the rates of the two pragmatic language measures (frequency of occurrence per minute) for children with WS: (1) extending a verbal interaction in response to a question and (2) extending a verbal interaction when a question had not been asked, after controlling for individual differences in non-verbal intellectual ability. We also addressed the question of whether the rates of these pragmatic behaviors were related to rate of pairing verbalizations with eye contact (secondary intersubjectivity) and/or expressive vocabulary ability, even after controlling for individual differences in non-verbal intellectual ability.

Our second set of questions was longitudinal. In particular, we sought to determine if individual differences in the pragmatic language abilities we measured were stable from the preschool period to the primary school period, after controlling for individual differences in non-verbal intellectual ability. We also considered the question of whether rate of pairing verbalizations with eye contact (secondary intersubjectivity) at age 4 years and/or expressive vocabulary ability at age 4 years (Time 1) predicted rate of extending a verbal interaction either in response to a question or in the absence of a question approximately 6 years later (Time 2 ) even after differences in non-verbal intellectual ability had been taken into account.

\section{MATERIALS AND METHODS PARTICIPANTS}

Participants were 14 children with genetically confirmed classiclength WS deletions ( 7 boys, 7 girls) for whom data were available at two time points. At Time 1 , mean CA was 4.30 years $(\mathrm{SD}=0.25$, range: 4.01-4.65). The children were re-assessed an average of 5.87 years later $(\mathrm{SD}=1.04$, range: $4.48-7.60)$. The children's mean $\mathrm{CA}$ at Time 2 was 10.18 years $(\mathrm{SD}=1.08$, range: $9.02-12.06)$. The racial/ethnic constitution of the sample was $7 \%$ White Hispanic and $93 \%$ White Non-Hispanic.

\section{MEASURES \\ Differential Ability Scales}

The Differential Ability Scales (DAS) is an individually administered standardized measure of verbal, non-verbal reasoning, and spatial (visuospatial construction) abilities that yields a General Conceptual Ability (GCA; similar to IQ) standard score (SS) and several cluster SSs. The mean for the general population is 100 
with a SD of 15 for both the GCA and the cluster SSs. Participants completed the DAS-Preschool Version (DAS; Elliott, 1990) at Time 1 and the DAS-II School-Age Version (DAS-II; Elliott, 2007) at Time 2. Non-verbal intellectual ability was measured by the DAS-Preschool Nonverbal cluster SS at Time 1; the subtests included in this cluster measure non-verbal reasoning and visuospatial construction. Non-verbal intellectual ability at Time 2 was measured by the DAS-II Special Nonverbal Composite (SNC) SS, which is based on performance on the subtests included in the Nonverbal Reasoning and Spatial clusters.

\section{Expressive Vocabulary Test}

The Expressive Vocabulary Test (EVT) is an individually administered standardized measure of expressive vocabulary ability. The mean for the general population is 100 with a SD of 15. Participants completed the EVT (Williams, 1997) at Time 1 and the EVT-2 (Williams, 2007) at Time 2.

\section{Pragmatic Language Samples}

The children's language during two spontaneous interactions with an adult, one at each time point, was transcribed and coded. At Time 1, each child participated in a 30-min play session with his or her mother in a laboratory playroom equipped with developmentally appropriate toys. At Time 2, each child participated in a 7-min conversation with a familiar researcher who was instructed to attempt to maintain a conversation with the child for the entire $7 \mathrm{~min}$ period. The researcher was given a list of suggested topics to introduce if she was unable to maintain the conversation by following the child's lead. The videotapes of the Pragmatic Language Samples were transcribed by trained research assistants and marked to indicate the presence of any pauses lasting $3 \mathrm{~s}$ or longer. Two research assistants checked the original transcript against the original video recording and made any changes necessary to arrive at a consensus transcript.

\section{PROCEDURE}

At both time points, children completed a battery of cognitive and language assessments including an assessment of intellectual abilities (DAS) and an assessment of expressive vocabulary (EVT). These measures were administered according to the test authors' instructions and were usually completed within a day of the measure of pragmatic language ability (Play session at Time 1 and Conversation at Time 2). The Pragmatic Language Samples were coded using the procedure described in the next section.

\section{TRANSCRIPT CODING}

Children's verbalizations during the Pragmatic Language Samples at both time points were coded for two pragmatic language variables: ExtendS (statements that the child made - not in response to adult questions - that served to appropriately extend the conversation) and ExtendQ (statements or questions that the child produced - in response to adult questions - that served to appropriately extend the conversation). In addition, children's EyeContact (utterances produced by the child that were accompanied by eye contact with the conversational partner) was coded at Time 1 as a measure of early secondary intersubjectivity. The coding system for each of these variables is described below.

\section{ExtendS}

Each statement that (1) the child produced in response to a statement made by the adult, (2) the child provided following a pause of more than $3 \mathrm{~s}$, or (3) if the child already held the floor, extended the conversation by adding new information was coded "yes" or "no." Children's statements were coded "yes" if they added new information to the interaction and did not fit either of the categories below. Statements were coded "no" if they did not add new information or they fit either of the following categories:

1. The child's response pertained to an inappropriate topic (e.g., personal bodily functions).

2. The child's statement insulted either the adult or a third party.

For each child at each time point, the variable ExtendS represented the rate per minute of statements (not made in response to a question) produced by the child that were coded "yes." This variable was calculated by dividing the number of statements (not made in response to a question) produced by the child that were coded "yes" by the length of the Pragmatic Language Sample (in minutes). High agreement was observed for both Time 1 (percentage of agreement $=92 \%, \kappa=0.83$ ) and Time 2 (percentage of agreement $=91 \%, \kappa=0.78)$.

\section{ExtendQ}

Each verbal response to an adult question that the child produced was coded "yes" or "no." Responses were coded "yes" if they either added appropriate information to the interaction beyond what was directly requested or if they both responded to the adult's question and included as part of their response an appropriate question directed toward the adult. Responses to questions were coded "no" if they fit any of the following categories:

1. The child's response pertained to an inappropriate topic (e.g., personal bodily functions).

2. The child's response insulted either the adult or a third party.

3. The child did not answer the adult's question within $4 \mathrm{~s}$.

4. The child ignored the question asked by the adult and produced an unrelated utterance.

5. The child's response included a question which he or she had previously asked multiple times and to which the adult had responded at least three times. The child's response was coded "yes" the first three times he or she asked a particular question and the adult answered the question. If the child continued to ask the same question even after the adult answered the question three times, subsequent repetitions of the question were coded "no." For example, if the child asked the same question five times (and the adult answered all five times), the first three times were coded "yes" and the last two were coded "no."

For each child at each time point, the variable ExtendQ represented the rate per minute of the child's responses to adult questions that were coded "yes." This variable was calculated by dividing the number of the child's responses to adult questions that were coded "yes" by the length of the Pragmatic Language Sample (in minutes). High agreement was observed for ExtendQ at both Time 1 (percentage of agreement $=98 \%, \kappa=0.74$ ) and Time 2 (percentage of agreement $=95 \%, \kappa=0.69$ ). 


\section{EyeContact}

At Time 1, each utterance produced by the child was coded "yes" or "no." Children's utterances were coded "yes" if the child made eye contact with the adult at any point during the verbalization and the utterance did not fit in either of the categories below. Utterances were coded "no" if the child did not make eye contact with the adult at any point during the utterance or if the utterance fit in either of the following categories:

1. The child's utterance only included a sound effect (e.g., eating or drinking noises, animal sounds).

2. The child's utterance was completely unintelligible.

For each child at each time point, the variable EyeContact represented the rate per minute of utterances produced by the child that were coded "yes." This variable was calculated by dividing the number of utterances produced by the child that were coded "yes" by the length of the Pragmatic Language Sample (in minutes). High agreement was observed for EyeContact at Time 1 (percentage of agreement $=95 \%, \kappa=0.98$ ). This variable could not be coded at Time 2, since the Pragmatic Language Sample at Time 2 was a dyadic interaction instead of a triadic interaction.

\section{RESULTS}

\section{PRAGMATIC ABILITY AT TIME 1}

Descriptive statistics for performance on the standardized assessments at Time 1 are reported in Table 1. Relative to prior reports of SSs for children with WS on these measures (e.g., Mervis and Morris, 2007), the mean level of performance of the present group of children was higher. The variability among children was at or above the typical level.

Descriptive statistics for the variables computed from the Pragmatic Language Sample at Time 1 are reported in Table 2. As both the assessment SSs and the variables from the Time 1 Pragmatic Language Sample met the necessary statistical assumptions for use of parametric analyses, Pearson correlations were used to compute relations among the dependent variables. Bivariate correlations of non-verbal intellectual ability with the pragmatic language variables, the secondary intersubjectivity variable, and expressive vocabulary ability at Time 1 are shown in Table 3 $\left(\alpha_{\mathrm{fw}}=0.0125\right)$. As a priori positive relations were predicted for all analyses conducted as part of this project, one-tailed tests were

Table 1 | Descriptive statistics for standardized assessment performance (standard scores) as a function of time point.

\begin{tabular}{llllllll}
\hline Measure & \multicolumn{3}{c}{ Time 1 } & & \multicolumn{3}{c}{ Time 2 } \\
\cline { 2 - 3 } \cline { 7 - 9 } & $\boldsymbol{M}$ & SD & Range & & $\boldsymbol{M}$ & SD & Range \\
\hline DAS GCA & 72.69 & 15.88 & $44-92$ & 67.36 & 13.00 & $43-91$ \\
DAS Nonverbal/SNC & 69.77 & 15.95 & $43-92$ & & 63.07 & 13.05 & $41-86$ \\
EVT SS & 91.57 & 21.86 & $40-116$ & & 85.79 & 16.03 & $58-112$
\end{tabular}

Time 1 assessments: DAS GCA, DAS Nonverbal cluster, EVT. Time 2 assessments: DAS-II GCA, DAS-II Special Nonverbal Composite (SNC), EVT-2. used throughout. As indicated in Table 3, non-verbal intellectual ability was significantly and strongly related to expressive vocabulary ability and marginally related to ExtendS. To control for individual differences in non-verbal intellectual ability, we computed partial correlations for the remaining Time 1 analyses, controlling for DAS Nonverbal cluster SS. The first set of partial correlations examined relations among EyeContact, ExtendS, and ExtendQ at Time $1\left(\alpha_{\mathrm{fw}}=0.025\right)$. At Time 1, EyeContact was significantly related both to ExtendS $(r=0.63, p=0.01)$ and to ExtendQ $(r=0.68, p=0.008)$ even after the effects of nonverbal intellectual ability were controlled. The correlation between ExtendS and ExtendQ was also significant $(r=0.80, p=0.001)$ even after controlling for non-verbal intellectual ability. Partial correlations were also computed to determine the relation between the two pragmatic language variables (ExtendS and ExtendQ) and expressive vocabulary ability (EVT SS). Results indicated that even after controlling for non-verbal intellectual ability, both ExtendS $(r=0.52, p=0.04)$ and ExtendQ $(r=0.53, p=0.04)$ were significantly correlated with EVT SS.

\section{PRAGMATIC ABILITY AT TIME 2}

Descriptive statistics for performance on the standardized assessments at Time 2 are also reported in Table $\mathbf{1}$. The children's SSs were slightly higher than previously reported for children with WS (e.g., Mervis and John, 2010) but evidenced the expected amount of variability.

Table 2 | Descriptive statistics for rates of Pragmatic Language Sample variables as a function of time point.

\begin{tabular}{|c|c|c|c|c|c|c|}
\hline \multirow{2}{*}{$\begin{array}{l}\text { Coded behavior } \\
\text { Measure }\end{array}$} & \multicolumn{3}{|c|}{ Time 1} & \multicolumn{3}{|c|}{ Time 2} \\
\hline & $M$ & SD & Range & $M$ & SD & Range \\
\hline \multicolumn{7}{|c|}{ SUCCESS RATE (NUMBER OFYES CODES PER MINUTE) } \\
\hline EyeContact & 1.59 & 1.06 & $0-3.80$ & - & - & - \\
\hline ExtendS & 1.47 & 0.84 & $0-3.20$ & 1.00 & 0.81 & $0-3.14$ \\
\hline ExtendQ & 0.32 & 0.26 & $0-0.90$ & 0.59 & 0.48 & $0-1.57$ \\
\hline \multicolumn{7}{|c|}{ FAILURE RATE (NUMBER OF NO CODES PER MINUTE) } \\
\hline EyeContact & 6.23 & 3.11 & $0.10-12.33$ & - & - & - \\
\hline ExtendS & 1.61 & 0.75 & $0-3.00$ & 1.20 & 0.61 & $0-2.00$ \\
\hline ExtendQ & 3.23 & 0.96 & $1.70-5.03$ & 6.44 & 1.77 & $3.43-8.86$ \\
\hline
\end{tabular}

Table 3 | Bivariate correlations of non-verbal intellectual ability with pragmatic language variables, secondary intersubjectivity, and expressive vocabulary ability as a function of time point.

\begin{tabular}{lll}
\hline Measure & Time 1 & Time 2 \\
\hline EyeContact & 0.13 & - \\
ExtendS & $0.52^{*}$ & $0.53^{*}$ \\
ExtendQ & 0.40 & 0.25 \\
EVT & $0.86^{* * *}$ & $0.76^{* *}$ \\
\hline
\end{tabular}

Time 1: Non-verbal intellectual ability: DAS Nonverbal cluster SS, Expressive vocabulary ability: EVT SS. Time 2: Non-verbal intellectual ability: DAS-II SNC, Expressive vocabulary ability: EVT-2 SS. ${ }^{*} p \leq 0.05,{ }^{* *} p \leq 0.001$. 
Descriptive statistics for performance on the variables computed from the Time 2 Pragmatic Language Sample are reported in Table 2. Data from the Time 2 standardized assessments and the pragmatic language variable ExtendQ met the necessary statistical assumptions for use of parametric analyses. As the distribution for ExtendS violated the parametric assumption of normality, a logarithmic transformation was applied. Bivariate correlations of non-verbal intellectual ability with the pragmatic language variables and expressive vocabulary ability at Time 2 also are shown in Table 3 ( $\left.\alpha_{\mathrm{fw}}=0.0125\right)$. Non-verbal intellectual ability was significantly and strongly related to expressive vocabulary ability and marginally related to ExtendS. To control for individual differences in non-verbal intellectual ability, we computed partial correlations for the remaining Time 2 analyses, controlling for DAS-II SNC. There was a significant correlation between logExtendS and ExtendQ $(r=0.59, p=0.02)$ after controlling for the effects of non-verbal intellectual ability. Partial correlations were also computed to determine the relation between the two pragmatic language variables and expressive vocabulary ability (EVT-2 SS) at Time 2 after controlling for the effects of non-verbal intellectual ability. Neither correlation was significant (logExtendS: $r=0.29$; ExtendQ: $r=0.19$ ).

\section{RELATIONS ACROSS TIME 1 AND TIME 2}

To examine the stability of individual differences in pragmatic language ability across the two time points after controlling for non-verbal intellectual ability at both time points, one-tailed Pearson correlation coefficients were computed controlling for both DAS Nonverbal SS and DAS-II School-Age SNC. Results indicated that Time 1 ExtendQ was significantly correlated with Time 2 ExtendQ $(r=0.64, p=0.02)$. The correlation between ExtendS at Time 1 and Time $2(r=0.26, p=0.23)$ was not significant. One-tailed partial correlation coefficients were also computed to determine if Time 1 EyeContact predicted Time 2 logExtendS or Time 2 ExtendQ. Results indicated that after controlling for nonverbal intellectual ability at both time points, Time 1 EyeContact predicted Time 2 ExtendQ $(r=0.72, p=0.01)$ but did not predict Time $2 \operatorname{logExtendS}(r=0.23, p=0.26)$. Finally, one-tailed Pearson correlation coefficients were also computed controlling for non-verbal intellectual ability at both Time 1 and Time 2 to determine if expressive vocabulary ability (EVT SS) at Time 1 predicted Time 2 logExtendS or Time 2 ExtendQ. Neither correlation was significant (ExtendQ: $r=0.15, p=0.31$, logExtendS: $r=-0.18$, $p=0.34)$.

\section{DISCUSSION}

Considerable attention has been drawn to the WS social phenotype due to its seemingly paradoxical nature. Although children with WS are described as highly gregarious and friendly, they have considerable difficulty establishing and maintaining friendships (e.g., Dilts et al., 1990; Gosch and Pankau, 1997; Dykens and Rosner, 1999; Sullivan et al., 2003). Similarly, even though children with WS demonstrate relative strengths in the structural aspects of language and in concrete vocabulary, they typically have difficulties with the pragmatic aspects of language (see Mervis and Becerra, 2007; Mervis and John, 2010 for review). These findings from prior research serve as a reminder that successful navigation of the social world requires more than just an interest in interacting with others, a relatively good vocabulary, and the ability to produce grammatical sentences; it also depends on a complex interweaving of cognitive, affective, and personality factors. In the present study, we sought to contribute to the growing literature exploring the WS social phenotype by examining the relations (beyond those accounted for by non-verbal intellectual ability) between individual differences in the following abilities both concurrently and predictively: verbal extension of an ongoing social exchange by contributing new information (both in response to questions and in the absence of questions) and expressive vocabulary. In addition, we examined whether or not individual differences in the coordination of eye contact and a verbal utterance (secondary intersubjectivity) predicted pragmatic language ability an average of 6 years later. In the remainder of the Discussion, we first address our cross-sectional findings separately as a function of time point and then consider our longitudinal findings. We then focus on the implications of these findings, the limitations of the present study, and future directions.

\section{CONCURRENT RELATIONS}

At both Time 1 (age 4 years) and Time 2 (age 9-12 years), the ability to verbally contribute new information beyond that requested in response to a question was significantly related to the ability to verbally contribute new information in the absence of a question, even after the effects of non-verbal intellectual ability were controlled. Furthermore, at Time 1, expressive vocabulary ability was significantly positively correlated with individual differences in children's ability to contribute new information both in response to questions and in the absence of questions even after controlling for the effects of non-verbal intellectual ability. The positive relation between expressive vocabulary ability and rate of extending a conversation either in response to a question or in the absence of a question at Time 1, even after controlling for individual differences in non-verbal intellectual ability, may reflect the fact that at age 4 years, the vocabularies of many of the children were limited, making providing information beyond what was requested challenging. The partial correlations between expressive vocabulary ability and contributing new information to the conversation beyond what was requested were not significant at Time 2 . This difference from Time 1 may have been due to the fact that by school age the vocabularies of all of the children were adequate for contributing information beyond what was requested to an interaction.

At age 4 years, there also was a clear positive association between the rate of pairing verbalizations with eye contact (secondary intersubjectivity) and the rate of verbally adding information to a social interaction beyond what was required, both in response to a question and in the absence of a question, even after controlling for the effects of non-verbal intellectual ability. Although this study was the first to specifically examine the relation between use of eye contact when talking with another person (intersubjectivity) and pragmatic language ability in children with WS, many researchers have addressed the role of eye contact within social interactions involving TD children (e.g., Argyle and Cook, 1976). The content of a communicative interaction is comprised of more than just the words that are exchanged between partners; the surrounding 
context; and non-verbal behaviors exchanged between partners also significantly contribute to the content (Clark and Marshall, 1981; Clark, 1996; Richardson et al., 2009). Eye contact serves as a way of accessing this additional information. Pairing eye contact with a communicative act in triadic interactions has been described as secondary intersubjectivity, a demonstration of an awareness of shared mental states (e.g., Zlatev, 2008). It has been theorized that early deficits in secondary intersubjectivity negatively affect later communicative competence. For example, in the case of individuals who have autism spectrum disorders, early deficits are observed in many behaviors reflecting secondary intersubjectivity such as joint attention and intentionality (American Psychiatric Association, 2000); these early deficits have been hypothesized to lead to the later difficulties in pragmatic language that are observed in individuals who have autism spectrum disorders (Rogers, 1998). The associations we found between pairing utterances with eye contact to the communicative partner during play with toys (secondary intersubjectivity) and verbally providing new information within a social exchange (pragmatics) is consistent with the argument that intersubjectivity is related to pragmatic ability.

We were unable to examine the relation between secondary intersubjectivity and verbal provision of information beyond what was requested at age 9-12 years due to context differences between our Time 1 and Time 2 Pragmatic Language Samples. In particular, because the Time 2 Pragmatic Language Sample involved a dyadic interaction rather than a triadic interaction, coding the rate that children paired verbalizations with eye contact would result in a measure of primary intersubjectivity (relating/connecting to other people in dyadic interactions). Children with WS have been shown to use eye contact during dyadic interactions at similar levels or higher levels as mental-age matched TD children (Laing et al., 2002). In contrast, in triadic joint attention situations, children with WS use eye contact significantly less often than either mental-age matched TD children (Laing et al., 2002) or children with DS matched for CA, DQ, and expressive vocabulary size (Rowe et al., 2005). Thus, primary and secondary intersubjectivity cannot be used interchangeably as measures of intersubjectivity for children with WS. It is important that future studies examine whether or not individual differences in secondary intersubjectivity remain stable across time for children with WS. Furthermore, given what is known about the dissociation between primary and secondary intersubjectivity in WS, longitudinal studies are needed that include both contexts that require triadic joint attention and contexts that require primarily dyadic joint attention. Such studies would be important for enhancing our understanding of the developmental trajectory of the use of eye contact by children with WS and its relation to pragmatic language.

Examination of the relations between pragmatic language ability across the two time points indicated that the rate of providing information beyond what was requested in response to a question at age 4 years significantly predicted the rate of providing information beyond what was expected in response to a question an average of 5.87 years later, even after controlling for non-verbal intellectual ability at both time points. Expressive vocabulary ability at age 4 did not predict school-age pragmatic ability. However, the rate of utterances children produced that were paired with eye contact (secondary intersubjectivity) as preschoolers did significantly predict the rate of provision of information beyond what was requested in response to questions during the school-age years, even after controlling for non-verbal intellectual ability at both time points.

Studies of joint attention in TD children have demonstrated that early joint attention ability predicts both later language development and the development of theory of mind (e.g., Tomasello, 1995; Baldwin and Moses, 1996; Charman et al., 2000, 2003). In addition to the role of language ability in conversational success, the ability to take the perspective of another person, or theory of mind, is vital for success in communicative interactions. John et al. (2009) found that the theory of mind ability of children with WS significantly and independently contributed to the likelihood that message inadequacy would be verbalized when the speaker's request was ambiguous. In addition, theory of mind ability significantly and independently contributed to the likelihood of effectively verbalizing the nature of the problem encountered when the speaker's message was ambiguous or when the speaker referred to the referent using a word that was not in the child's vocabulary. As triadic joint attention ability is a precursor to theory of mind and is a measure of secondary intersubjectivity, it is not surprising that we found that, for children with WS, early secondary intersubjectivity ability predicted later aspects of pragmatic language ability. This finding provides further support for the argument that early secondary intersubjectivity is a contributing factor to the development of pragmatic language ability.

\section{IMPLICATIONS}

The present study is the first to show a predictive association between early secondary intersubjectivity and later pragmatic ability in children with WS and to demonstrate consistency in individual differences in aspects of pragmatic language ability across time beyond what would have been expected given individual differences in non-verbal intellectual ability. The purpose of communicative exchanges often goes beyond just the transfer of information between individuals; communicative exchanges involve connecting with another person (Zlatev, 2008). Our longitudinal finding that early secondary intersubjectivity ability predicted later pragmatic language ability even after controlling for individual differences in non-verbal intellectual ability strongly suggests that limitations in secondary intersubjectivity early in development contribute to later deficits in pragmatic language, providing a causal link between two key components of the WS social phenotype. Thus, this longitudinal finding provides clear evidence of an important link between prior cross-sectional findings of impairments in aspects of secondary intersubjectivity (triadic joint attention) in very young children with WS (e.g., Klein-Tasman et al., 2007; Lincoln et al., 2007) and impairments in pragmatic language in older children with WS (e.g., Udwin and Yule, 1990; Laws and Bishop, 2004; Stojanovik, 2006; Philofsky et al., 2007).

In light of these predictive associations, it is important that interventions be developed targeting secondary intersubjectivity in young children with WS. Furthermore, as previously stressed by Mervis and Becerra (2007; see also Mervis and John, 2010; Mervis and Velleman, 2011), parents, therapists, and teachers need to be vigilant to avoid being deceived by the relatively good 
expressive language of children with WS into assuming that their communicative skills are adequate for their developmental levels. Intervention programs such as the Early Start Denver Model (ESDM; Rogers and Dawson, 2010) that use a variety of techniques to directly address secondary intersubjectivity deficits provide a framework for targeting these skills in WS. Results of a randomized controlled trial of ESDM for children with autism spectrum disorders between 18 and 30 months of age indicated that 2 years after entering intervention children who received ESDM demonstrated significant improvements in IQ, adaptive behavior, and autism symptomology when compared to children who received intervention from community providers (Dawson et al., 2010). As discussed by Mervis and John (2010), the overlap in types of socio-communicative difficulties demonstrated by children with autism spectrum disorders and children with WS suggests that therapeutic approaches similar to ESDM will be appropriate and effective for children with WS, once modifications to account for differences in the behavioral phenotypes of children with WS as compared to children with autism spectrum disorders are made.

\section{LIMITATIONS AND FUTURE DIRECTIONS}

While the findings from the present study contribute to the growing literature exploring the WS social phenotype, we acknowledge some limitations. First, because the sample size was relatively small, we did not have adequate power to detect small to moderate effects. Second, because we did not include a contrast group, we were not able to address the question of whether the ability of children with WS to verbally contribute new information to a social interaction is similar to or different from that demonstrated by other groups with intellectual disability. We also were not able to address the generality of our longitudinal findings for children with other syndromes. The context in which the Time 1 Pragmatic Language Sample was collected (play with toys with the child's mother) differed from the context in which the Time 2 Pragmatic Language Sample was collected (conversation with a familiar adult, with no objects present), which may have reduced the extent of continuity of individual differences in pragmatic language abilities from age 4 years to age $9-12$ years.

More research examining the longitudinal trajectories of primary and secondary intersubjectivity and pragmatic language and their relations to non-verbal ability and language ability for children with WS is needed, ideally with multiple data points across a wide age range. An important focus for this research would be to determine if individual differences in secondary intersubjectivity, which are strongly related to individual differences in pragmatic

\section{REFERENCES}

American Academy of Pediatrics Committee on Genetics. (2001). Health care supervision for children with Williams syndrome. Pediatrics 107, 1192-1204.

American Psychiatric Association. (2000). Diagnostic and Statistical Manual of Mental Disorders 4th Edn, (Text Revision). Washington, DC: American Psychiatric Association.

Argyle, M., and Cook, M. (1976). Gaze and Mutual Gaze.
Cambridge: Cambridge University Press.

Baldwin, D. A., and Moses, L. J. (1996). The ontogeny of social information gathering. Child Dev. 67, 1915-1939.

Bishop, D. V. M. (1998). Development of the Children's Communication Checklist (CCC): a method for assessing the qualitative aspects of communication impairment in children. J. Child Psychol. Psychiatry 39, 879-891.

language abilities at age 4 years, are stable across time. In addition, it is important for future studies to examine the relations among primary intersubjectivity, secondary intersubjectivity, and pragmatic language ability over time. Finally, future studies (both cross-sectional and longitudinal) should also compare the pragmatic abilities of children with WS to those of matched children with other etiologies of intellectual disability to identify similarities and differences as a function of syndrome.

\section{CONCLUSION}

Over the past few decades, more and more interest has developed with regard to understanding the social phenotype associated with WS. Despite considerable interest in other people, children with WS demonstrate difficulty both with pragmatic language and with establishing and maintaining friendships. In the present study, we found that the ability to verbally contribute information beyond what was required in response to a question was significantly related to the ability to verbally contribute new information in the absence of a question during both the preschool years and the school-age years. During the preschool years, the ability to pair verbalizations with eye contact (secondary intersubjectivity) was related to the ability to verbally contribute information beyond the minimum expected within a social interaction. Finally, the ability to verbally contribute new information to a social interaction beyond what was required to answer a question and the ability to pair verbalizations with eye contact (secondary intersubjectivity) at age 4 years predicted the ability to verbally contribute new information beyond what was required to answer a question at age 9-12 years. Understanding the nature of the pragmatic abilities of children with WS over time and the relations of these abilities to social cognition, language ability, and non-verbal intellectual ability is a crucial step toward the development of interventions to address the socio-communicative difficulties evidenced by individuals with WS.

\section{ACKNOWLEDGMENTS}

This research was supported by grants R37 HD29957 from the National Institute of Child Health and Human Development and R01 NS35102 from the National Institute of Neurological Disorders and Stroke. We thank the children and their parents for their enthusiastic participation in our research. We also thank the many members of the Neurodevelopmental Sciences Laboratory who transcribed the play sessions and conversations for this project.

Bishop, D. V. M. (2002). The Children's Communication Checklist, 2nd Edn. London: Psychological Corporation.

Charman, T., Baron-Cohen, S., Swettenham, J., Baird, G., Drew, A., and Cox, A. (2000). Testing joint attention, imitation, and play as infancy precursors to language and theory of mind. Cogn. Dev. 15, 481-498.

Charman, T., Baron-Cohen, S., Swettenham, J., Baird, G., Drew, A., and Cox, A. (2003). Predicting language outcome in infants with autism and pervasive developmental disorder. Int. J. Lang. Commun. Disord. 38, 265-285.

Clark, H. H. (1996). Using Language. Cambridge: Cambridge University Press.

Clark, H. H., and Marshall, C. R. (1981). "Definite reference and mutual knowledge," in Perspectives on Socially Shared Cognition, eds A. K. Joshi, I. Webber, and S. D. Teasley (Washington, DC: American Psychological Association), 127-149. 
Constantino, J. N., and Gruber, C. P. (2005). Manual for Social Responsiveness Scale. Los Angeles, CA: Western Psychological Services.

Cowley, G. (2003). Girls, boys and autism. Newsweek 142, 42-50.

Davies, M., Udwin, O., and Howlin, P. (1998). Adults with Williams syndrome. Preliminary study of social, emotional and behavioural difficulties. Br. J. Psychiatry 172, 273-276.

Dawson, G., Rogers, S., Munson, J., Smith, M., Winter, J., Greenson, J., Donaldson, A., and Varley, J. (2010). Randomized, controlled trial of an intervention for toddlers with autism: the Early Start Denver Model. Pediatrics 125, e17-e23.

Dilts, C. V., Morris, C. A., and Leonard, C. O. (1990). Hypothesis for development of a behavioral phenotype in Williams syndrome. Am. J. Med. Genet. Suppl. 6, 126-131.

Dykens, E. M., and Rosner, B. A. (1999). Refining behavioral phenotypes: personality-motivation in Williams and Prader-Willi syndromes. Am. J. Ment. Retard. 104, 158-169.

Elliott, C. D. (1990). Differential Ability Scales. San Antonio, TX: Psychological Corporation.

Elliott, C. D. (2007). Differential Ability Scales, 2nd Edn. San Antonio, TX: Psychological Corporation.

Fidler, D. J., Hepburn, S., Most, D., and Philofsky, A. (2007). Emotional responsivity in young children with Williams syndrome. Am. J. Ment. Retard. 112, 194-206.

Fryns, J. P., Borghgraef, M., Volcke, P., and van den Berghe, H. (1991). Adults with Williams syndrome. Am. J. Med. Genet. 40, 253.

Gosch, A., and Pankau, R. (1997). Personality characteristics and behaviour problems in individuals of different ages with Williams syndrome. Dev. Med. Child Neurol. 39, 527-533.

Harmon, A., John, A. E., and Mervis, C. B. (2009). "Pragmatic language ability in children with Williams syndrome," in Poster presented at the Symposium on Research in Child Language Disorders. Madison, WI.

Hillier, L. W., Fulton, R. S., Fulton, L. A., Graves, T. A., Pepin, K. H., Wagner-McPherson, C., Layman, D., Maas, J., Jaeger, S., Walker, R., Wylie, K., Sekhon, M., Becker, M. C., O'Laughlin, M. D., Schaller, M. E., Fewell, G. A., Delehaunty, K. D., Miner, T. L., Nash, W. E., Cordes, M., Du, H., Sun, H., Edwards, J., Bradshaw-Cordum, H., Ali, J., Andrews, S., Isak, A., Vanbrunt,
A., Nguyen, C., Du, F., Lamar, B., Courtney, L., Kalicki, J., Ozersky, P., Bielicki, L., Scott, K., Holmes, A., Harkins, R., Harris, A., Strong, C. M., Hou, S., Tomlinson, C., DauphinKohlberg, S., Kozlowicz-Reilly, A., Leonard, S., Rohlfing, T., Rock, S. M., Tin-Wollam, A. M., Abbott, A., Minx, P., Maupin, R., Strowmatt, C., Latreille, P., Miller, N., Johnson, D., Murray, J., Woessner, J. P., Wendl, M. C., Yang, S. P., Schultz, B. R., Wallis, J. W., Spieth, J., Bieri, T. A., Nelson, J. O., Berkowicz, N., Wohldmann, P. E., Cook, L. L., Hickenbotham, M. T., Eldred, J., Williams, D., Bedell, J. A., Mardis, E. R., Clifton, S. W., Chissoe, S. L., Marra, M. A., Raymond, C., Haugen, E., Gillett, W., Zhou, Y., James, R., Phelps, K., Iadanoto, S., Bubb, K., Simms, E., Levy, R., Clendenning, J., Kaul, R., Kent, W. J., Furey, T. S., Baertsch, R. A., Brent, M. R., Keibler, E., Flicek, P., Bork, P., Suyama, M., Bailey, J. A., Portnoy, M. E., Torrents, D., Chinwalla, A. T., Gish, W. R., Eddy, S. R., McPherson, J. D., Olson, M. V., Eichler, E. E., Green, E. D., Waterston, R. H., and Wilson, R. K. (2003). The DNA sequence of chromosome 7. Nature 424, 157-164.

John, A. E., and Mervis, C. B. (2009). "The relation between theory of mind and language in children with Williams syndrome," Poster presented at the Symposium on Research in Child Language Disorders, Madison, WI.

John, A. E., and Mervis, C. B. (2010). Comprehension of the communicative intent behind pointing and gazing gestures by young children with Down syndrome and Williams syndrome. J. Speech Lang. Hear. Res. 53, 950-960.

John, A. E., Rowe, M. L., and Mervis, C. B. (2009). Referential communication skills of children with Williams syndrome: understanding when messages are not adequate. Am. J. Intellect. Dev. Disabil. 114, 85-99.

Jones, W., Bellugi, U., Lai, Z., Chiles, M., Reilly, J., Lincoln, A., and Adolphs, R. (2000). II. Hypersociability in Williams syndrome. J. Cogn. Neurosci. 12(Suppl. 1), 30-46.

Klein-Tasman, B. P., Li-Barber, K. T., and Magargee, E. T. (2011). Honing in on the social phenotype in Williams syndrome using multiple measures and multiple raters. J. Autism Dev. Disord. 41, 341-351.

Klein-Tasman, B. P., and Mervis, C. B. (2003). Distinctive personality characteristics of 8-, 9-, and 10-year-olds with Williams syndrome. Dev. Neuropsychol. 23 269-290.

Klein-Tasman, B. P., Mervis, C. B., Lord, C., and Phillips, K. (2007). Sociocommunicative deficits in young children with Williams syndrome: performance on the Autism Diagnostic Observation Schedule. Child Neuropsychol. 13, 444-467.

Laing, E., Butterworth, G., Ansari, D., Gsodl, M., Longhi, E., Panagiotaki, G., Paterson, S., and Karmiloff-Smith, A. (2002). Atypical development of language and social communication in toddlers with Williams syndrome. Dev. Sci. 5, 233-246.

Laws, G., and Bishop, D. (2004). Pragmatic language impairment and social deficits in Williams syndrome: a comparison with Down's syndrome and specific language impairment. Int. J. Lang. Commun. Disord. 39, 45-64.

Levy, D., Ronemus, M., Yamrom, B., Lee, Y. H., Leotta, A., Kendall, J., Marks, S., Lakshmi, B., Pai, D., Ye, K., Buja, A., Krieger, A., Yoon, S., Troge, J., Rodgers, L., Iossifov, I., and Wigler, M. (2011). Rare de novo and transmitted copy-number variation in autistic spectrum disorders. Neuron 70, 886-897.

Lincoln, A. J., Searcy, Y. M., Jones, W., and Lord, C. (2007). Social interaction behaviors discriminate young children with autism and Williams syndrome. J. Am. Acad. Child Adolesc. Psychiatry 46, 323-331.

Lord, C., Rutter, M., DiLavore, P. C., and Risi, S. (1999). Autism Diagnostic Observation Schedule. Los Angeles: Western Psychological Services.

Mervis, C. B., and Becerra, A. M. (2007). Language and communication development in Williams syndrome. Ment. Retard Dev. Disabil. Res. Rev. 13, 3-15.

Mervis, C. B., and Bertrand, J. (1993). "Acquisition of early object labels: the roles of operating principles and input," in Enhancing Children's Communication: Research Foundations for Intervention, eds A. P. Kaiser and D. B. Gray (Baltimore, MD: Brookes), 287-316.

Mervis, C. B., and Bertrand, J. (1997). "Developmental relations between cognition and language: evidence from Williams syndrome," in Research on Communication and Language Disorders: Contributions to Theories of Language Development, eds L. B. Adamson and M. A. Romski (Baltimore, MD: Brookes), 75-106.
Mervis, C. B., and John, A. E. (2010). Cognitive and behavioral characteristics of children with Williams syndrome: implications for intervention approaches. Am. J. Med. Genet. C Semin. Med. Genet. 154C, 229-248.

Mervis, C. B., and Morris, C. A. (2007). "Williams syndrome," in Neurogenetic Developmental Disorders: Variation of Manifestation in Childhood eds M. M. M. Mazzocco and J. L. Ross (Cambridge: MIT Press), 199-262.

Mervis, C. B., Morris, C. A., KleinTasman, B. P., Bertrand, J., Kwitny, S., Appelbaum, L. G., and Rice, C. E. (2003). Attentional characteristics of infants and toddlers with Williams syndrome during triadic interactions. Dev. Neuropsychol. 23, 243-268.

Mervis, C. B., Robinson, B. F., Bertrand, J., Morris, C. A., Klein-Tasman, B. P., and Armstrong, S. C. (2000). The Williams syndrome cognitive profile. Brain Cogn. 44, 604-628.

Mervis, C. B., and Velleman, S. L. (2011). Children with Williams syndrome: language, cognitive, and behavioral characteristics and their implications for intervention. Perspect. Lang. Learn. Educ. 18, 98-107.

Morris, C. A. (2006). "The dysmorphology, genetics, and natural history of Williams-Beuren syndrome," in Williams-Beuren Syndrome: Research, Evaluation and Treatment, eds C. A. Morris, H. M. Lenhoff, and P. P. Wang (Baltimore: Johns Hopkins University Press), 3 17

Peregrine, E., Rowe, M. L., and Mervis, C. B. (2005). "Pragmatic language difficulties in children with Williams syndrome," in Poster presented at the meeting of the Society for Research in Child Development, Atlanta.

Philofsky, A., Fidler, D. J., and Hepburn, S. (2007). Pragmatic language profiles of school-age children with autism spectrum disorders and Williams syndrome. Am. J. Speech Lang. Pathol. 16, 368-380.

Richardson, D. C., Dale, R., and Tomlinson, J. M. (2009). Conversation, gaze coordination, and beliefs about visual context. Cogn. Sci. 33, 1468-1482.

Rogers, S. J. (1998). Neuropsychology of autism in young children and its implications for early intervention. Ment. Retard Dev. Disabil. Res. Rev. 4, 104-112.

Rogers, S. J., and Dawson, G. (2010). Early Start Denver Model for Young Children with Autism: Promoting 
Language, Learning, and Engagement. New York: Guilford Press.

Rowe, M. L., Peregrine, E., and Mervis, C. B. (2005). "Communicative development in toddlers with Williams syndrome," in Poster presented at the meeting of the Society for Research in Child Development, Atlanta, GA.

Stojanovik, V. (2006). Social interaction deficits and conversational inadequacy in Williams syndrome. J. Neurolinguistics $19,157-173$.

Strømme, P., Bjørnstad, P. G., and Ramstad, K. (2002). Prevalence estimation of Williams syndrome. J. Child Neuropsychol. 17, 269-271.

Sullivan, K., Winner, E., and TagerFlusberg, H. (2003). Can adolescents with Williams syndrome tell the difference between lies and jokes? Dev. Neuropsychol. 23, 85-104.

Tager-Flusberg, H., and Sullivan, K. (2000). A componential view of theory of mind: evidence from Williams syndrome. Cognition 76, 59-89.

Tager-Flusberg, H., Sullivan, K., and Boshart, J. (1997). Executive functions and performance on false belief tasks. Dev. Neuropsychol. 13, 487-493.

Thompson, R. A. (2006). "The development of the person: social understanding, relationships, conscience, and self," in Handbook of child psychology, Vol. 3, eds N. Eisenberg, W. Damon, and R. M. Lermer (Hoboken, NJ: Wiley), 24-98.

Tomasello, M. (ed.). (1995). Joint Attention as Social Cognition. Hillsdale, NJ: Erlbaum.

Udwin, O. (1990). A survey of adults with Williams syndrome and idiopathic infantile hypercalcaemia. Dev. Med. Child Neurol. 32, 129-141.
Udwin, O., and Yule, W. (1990). Expressive language of children with Williams syndrome. Am. J. Med. Genet. Suppl. 6, 108-114.

Udwin, O., and Yule, W. (1991). A cognitive and behavioral phenotype in Williams syndrome. J. Clin. Exp. Neuropsychol. 13, 232-244.

Williams, K. T. (1997). Expressive Vocabulary Test. Minneapolis, MN: Pearson Assessments.

Williams, K. T. (2007). Expressive Vocabulary Test, 2nd Edn. Minneapolis, $\mathrm{MN}$ : Pearson Assessments.

Zlatev, J. (ed.). (2008). The Shared Mind: Perspectives on Inter Subjectivity. Amsterdam: John Benjamin Publishing Co.

Conflict of Interest Statement: The authors declare that the research was conducted in the absence of any commercial or financial relationships that could be construed as a potential conflict of interest.

Received: 28 February 2012; paper pending published: 19 March 2012; accepted: 29 May 2012; published online: 18 June 2012.

Citation: John AE, Dobson LA, Thomas $L E$ and Mervis CB (2012) Pragmatic abilities of children with Williams syndrome: a longitudinal examination. Front. Psychology 3:199. doi: 10.3389/fpsyg.2012.00199

This article was submitted to Frontiers in Developmental Psychology, a specialty of Frontiers in Psychology.

Copyright (C) 2012 John, Dobson, Thomas and Mervis. This is an open-access article distributed under the terms of the Creative Commons Attribution Non Commercial License, which permits noncommercial use, distribution, and reproduction in other forums, provided the original authors and source are credited. 